\title{
$1470 \mathrm{~nm}$ Diode Laser Enucleation vs Plasmakinetic Resection of the Prostate for Benign Prostatic Hyperplasia: A Randomized Study
}

\author{
Jun Zhang, MM, Xilong Wang, MM, Yanbin Zhang, BM, Chaoliang Shi, BM, Minqi Tu, BM, and Guowei Shi, MD
}

\begin{abstract}
Objective: The purpose of the current work was to comparatively assess $1470 \mathrm{~nm}$ diode laser enucleation of the prostate (DiLEP) and plasmakinetic resection of the prostate (PKRP) for treating benign prostatic hyperplasia (BPH). Patients and Methods: From January 2016 to March 2017, 157 individuals with bladder outflow obstruction caused by BPH were randomized to DiLEP and PKRP groups, for prospective analysis. Of these, 152 cases were evaluated before operation and at 3, 6, and 12 months postsurgery. Patient baseline properties, presurgery data, and postsurgical outcomes were comparatively assessed, as well as complications.

Results: There were no significant preoperative differences between surgical groups. DiLEP-treated cases showed remarkable reduced operative time, postsurgical bladder irrigation time, catheterization duration, and hospital stay compared with the PKRP group $(P<0.001)$. Hemoglobin amount decrease was markedly less pronounced after DiLEP $(P=0.004)$. However, no patients needed blood transfusion in either group. The decrease in sodium level showed no marked differences between the DiLEP and PKRP groups $(P=0.380)$. In addition, complications were comparable and no significant differences in both groups. At 3, 6, and 12 months, International Prostate Symptom Score (IPSS), quality of life (QoL), maximum flow rate (Qmax), and postvoid residual (PVR) were similar in both groups $(P>0.05)$.

Conclusions: DiLEP and PKRP are similar in efficacy and safety for relieving obstruction and low urinary tract symptoms. Compared with PKRP, DiLEP has decreased risk of hemorrhage, operative time, bladder irrigation time, catheterization duration, and hospital stay. However, IPSS, QoL, Qmax, and PVR were similar for both procedures within 12 postoperative months.
\end{abstract}

Keywords: benign prostatic hyperplasia, $1470 \mathrm{~nm}$ diode laser, enucleation, TURP

Introduction

B ENIGN PROSTATIC HYPERPLASIA (BPH) constitutes the major etiology of lower urinary tract symptoms (LUTS) in male individuals aged $>50$ years. Transurethral resection of the prostate (TURP) represents the gold standard in the operative management of BPH, with demonstrated safety, efficacy, and durability. ${ }^{1}$ Although important technologic advances in the last few decades have decreased surgeryrelated undesirable events, complications, including bleeding $(0.3 \%)$, capsular perforation $(0.1 \%)$, transfusion $(2 \%)$, and transurethral resection syndrome (TURS; $0.8 \%$ ), remain a great concern. ${ }^{2}$ Therefore, a novel minimally invasive technology has been proposed, with laser surgery considered the new standard. ${ }^{3}$
The first laser enucleation of the prostate for BPH was described by Fraundorfer and Gilling in $1998 .{ }^{4}$ Then, a variety of laser types, for example, holmium, thulium, potassium-titanyl-phosphate (KTP), and diode lasers, have been used for treating $\mathrm{BPH}^{5-8}$ The first diode laser had approval from the U.S. Food and Drug Administration (FDA) in 2007, with wide use thanks to remarkable tissue vaporization capacity and a great coagulation property. ${ }^{9}$ This laser operated at $1470 \mathrm{~nm}$ absorbed by both water and hemoglobin. In addition, the $1470 \mathrm{~nm}$ diode laser remarkably improves International Prostate Symptom Score (IPSS), quality of life (QoL), maximum flow rate (Qmax), and postvoid residual (PVR). ${ }^{10}$

To assess the $1470 \mathrm{~nm}$ diode laser enucleation of the prostate (DiLEP) for BPH, a prospective randomized clinical

Department of Urology, The Fifth People's Hospital of Shanghai, Fudan University, Shanghai, P.R. China.

(C) Jun Zhang, et al. 2019; Published by Mary Ann Liebert, Inc. This Open Access article is distributed under the terms of the Creative Commons License (http://creativecommons.org/licenses/by/4.0), which permits unrestricted use, distribution, and reproduction in any medium, provided the original work is properly cited. 
trial (RCT) with 12-month follow-up was carried out, with plasmakinetic bipolar resection of the prostate (PKRP) as a control procedure.

\section{Patients and Methods}

\section{Patients}

The current prospective, single-blinded RCT was conducted between January 2016 and March 2017 in our department; all cases with LUTS due to BPH with indication ${ }^{11}$ for endosurgical treatment were invited to participate in this clinical study. In all, 157 cases were randomized to the DiLEP (79) and PKRP (78) groups after ethics committee approval and written informed consent from patients. Grouping strategy was performed with sequential numbering and sealed envelopes. The patients were assigned envelopes by a computerized random number generator. Cases eligible for surgical treatment of $\mathrm{BPH}$, with a prostate volume less than or equal to $80 \mathrm{~mL}$, were included. Exclusion criteria were as follows: neurogenic bladder, urethral stricture, prostate carcinoma, and a history of urethral or prostate surgery. Finally, 152 patients (DiLEP 76 vs PKRP 76) with complete followup data were analyzed (Fig. 1).

\section{Assessment parameters}

All patients underwent urine analysis, digital rectal examination, serum prostate-specific antigen (PSA) level evaluation, and urodynamic examination. The prostate was assessed for size by transrectal ultrasonography (TRUS), with the volume obtained as height $\times$ length $\times$ width $\times \pi / 6$. If prostate cancer was suspected, TRUS-guided biopsy was carried out for confirmation. Patient baseline properties were obtained, including age, prostate volume, PSA, Qmax, PVR, IPSS, and the QoL. Hemoglobin and serum sodium were measured before and 2 hours postsurgery. Perioperative indexes as well as peri- and postsurgical complications were recorded, for example, operative time, serum sodium and hemoglobin level changes, postsurgery irrigation time, catheterization time, hospitalization duration, blood transfusion requirement, TURS, capsular perforation, and urethral stricture. Follow-up was performed at 3,6, and 12 postoperative months. Clinical outcomes, including IPSS, QoL, Qmax and PVR, were assessed at each follow-up.

\section{Instruments and surgical procedures}

All operative procedures were carried out by one chief surgeon experienced in DiLEP and PKRP. The patients underwent detailed preoperative risk evaluation and were administered epidural or general anesthesia for surgical procedures in the lithotomy position. Major equipment for surgery included the following: Gyrus Plasmakinetic superpulse system generator (Gyrus), $120 \mathrm{~W} 1470 \mathrm{~nm}$ diode semiconductor laser generator (Miracle Laser, Wuhan, China), Olympus $12^{\circ}$ resectoscope (Olympus), and Hawk $30^{\circ}$ laser operator and morcellator system (Hawk, Hangzhou, China).

\section{Surgical technique}

The generator settings for PKRP were 180 and $100 \mathrm{~W}$ for cutting and coagulation, respectively. Physiologic saline was used for irrigation. In the PKRP group, bipolar TURP was performed routinely using a $26 \mathrm{~F}$ Olympus continuous irrigation resectoscope. Incision depth was close to the surgical capsule.

The $1470 \mathrm{~nm}$ diode laser generator for DiLEP had settings of 120 and $30 \mathrm{~W}$ for vaporization and coagulation, respectively. A $26 \mathrm{~F}$ resectoscope was inserted in the bladder under video assistance by an endosurgical device. The incision was started proximal to the verumontanum to the bladder neck at $5 \mathrm{o}^{\prime}$ clock and $7 \mathrm{o}^{\prime}$ clock positions, with the urethral mucosa
FIG. 1. In all, 157 cases were randomized to the DiLEP (79) and PKRP (78) groups after ethics committee approval and written informed consent from patients. Finally, 152 patients (DiLEP 76 vs PKRP 76) with complete follow-up data were analyzed. DiLEP $=$ diode laser enucleation of the prostate; $\mathrm{PKRP}=$ plasmakinetic resection of the prostate.

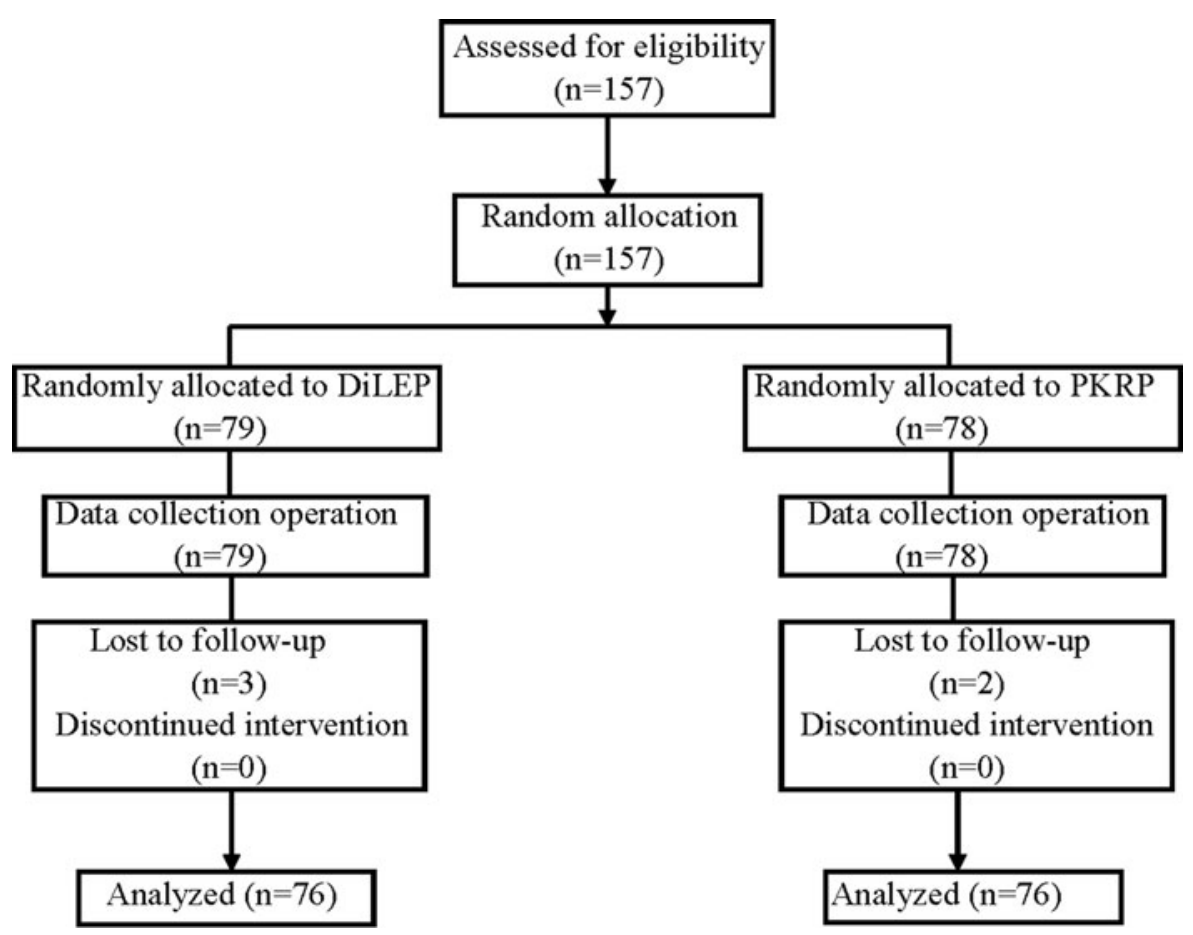




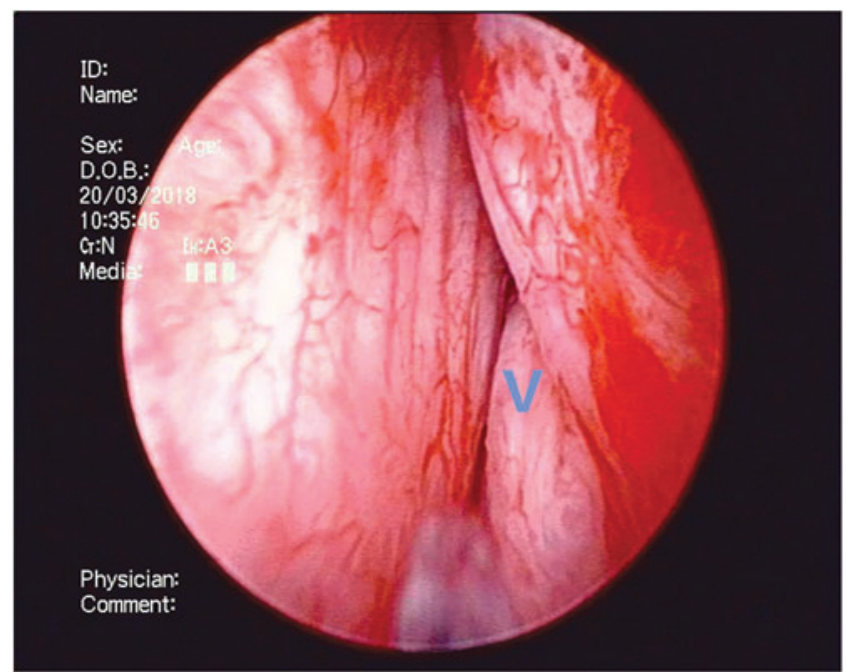

FIG. 2. The resectoscope is placed into the bladder (B) to observe the urethra and estimate the range between bladder neck and verumontanum (V). The ureteral orifices and the shape of the prostate are also assessed.

incised to surgical capsule's level. The middle lobe was enucleated retrogradely off the bladder neck. The lateral lobes were similarly enucleated along the capsule, moving clockwise (right lateral lobe) or counterclockwise (left lateral lobe). In case of bleeding, the laser beam was redirected to the specific area for hemostasis with $30 \mathrm{~W}$ power. Finally, the adenomas in the bladder were removed by the morcellator system (Figs. 2-7). Complete enucleation should not be performed in all cases; patients with a small prostate gland were treated by vaporesection. Therefore, a combination of enucleation and vaporesection was performed. At the end of both procedures, a $22 \mathrm{~F}$ three-way Foley catheter was inserted, and physiologic saline irrigation was continually administered.

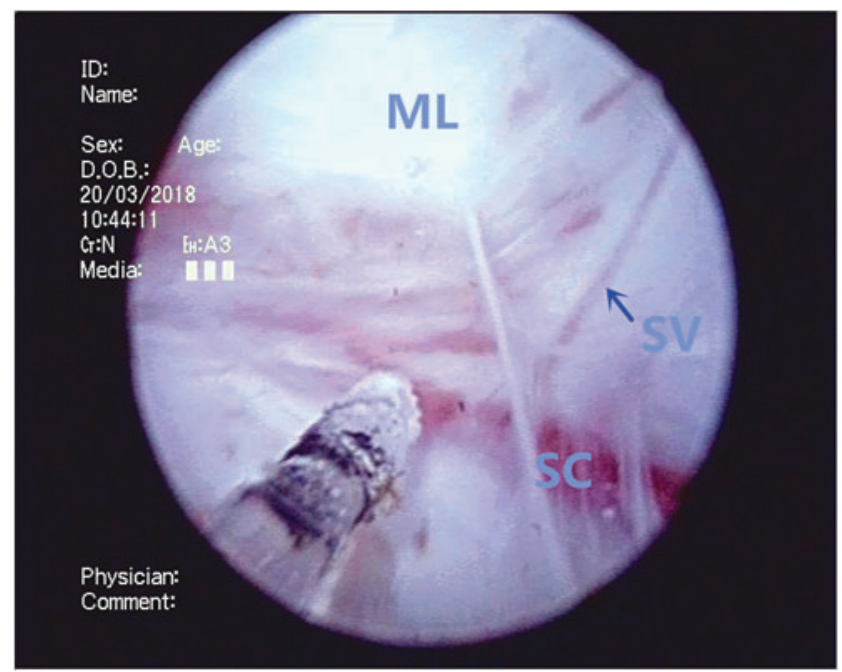

FIG. 3. The middle lobe (ML) is enucleated from the surgical capsule (SC) by the diode laser. Denuded supply vessels (SV) on the capsule surface are identified. In case of bleeding, the laser beam is redirected to the specific area for hemostasis.

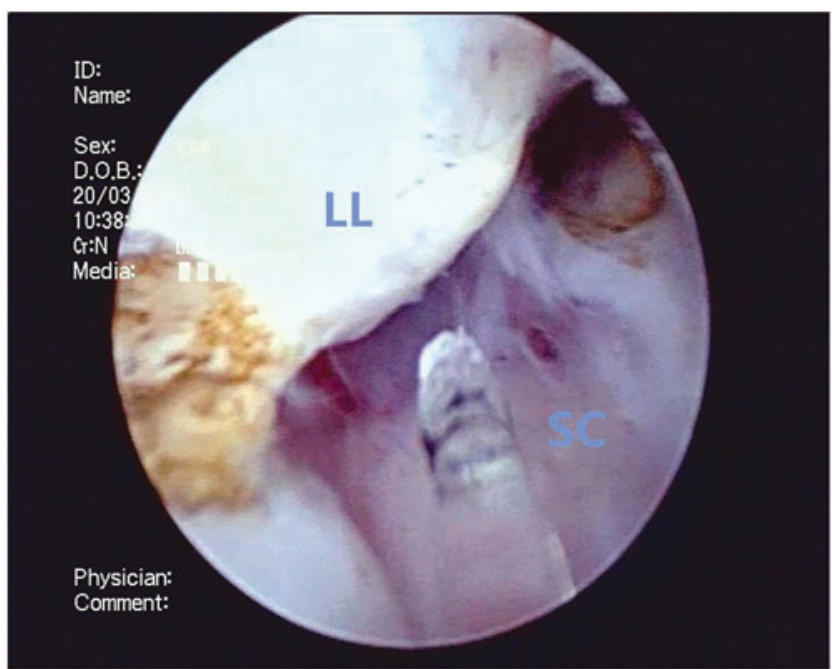

FIG. 4. The left lateral lobe (LL) is similarly enucleated along the surgical capsule, moving in a counterclockwise position.

\section{Statistical analysis}

All measurement data are mean \pm standard deviation, and were assessed by Student's $t$-test with Statistical Package for the Social Sciences (SPSS; v19.0). Postsurgery adverse events were assessed by the two-tailed chi-squared test. $P<0.05$ indicated statistical significance.

\section{Results}

Patient baseline features in both DiLEP and PKRP groups (Table 1) were not significantly different in any aspect. Perioperative indexes are summarized in Table 2. Hyponatremia was not observed in this study, and serum sodium decreases in both groups were similar $(P=0.380)$. In addition, weights of resected specimen were comparable between groups $(P=0.448)$. However, in comparison with PKRP

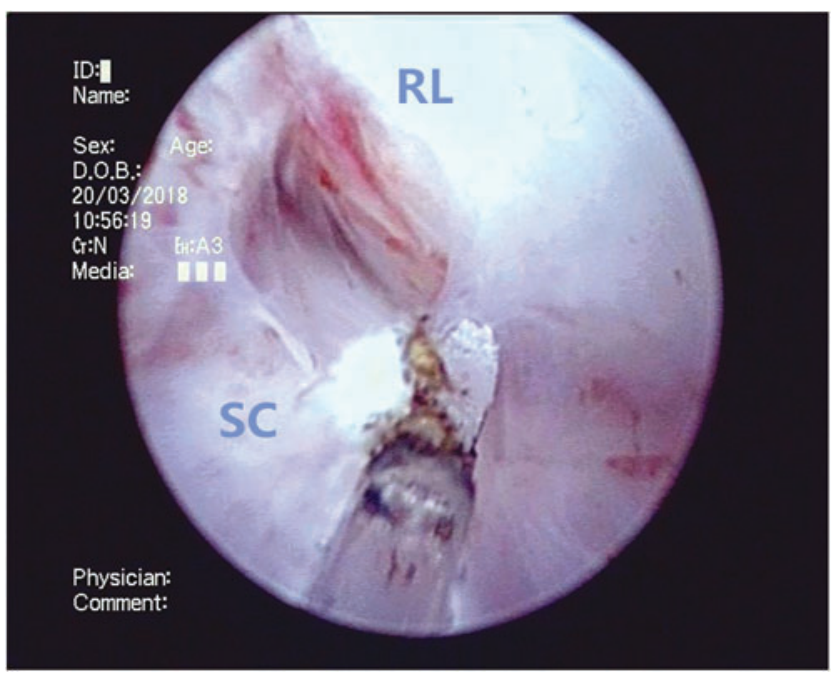

FIG. 5. The right lateral lobe (RL) is also similarly enucleated along the surgical capsule, moving in a clockwise position. 


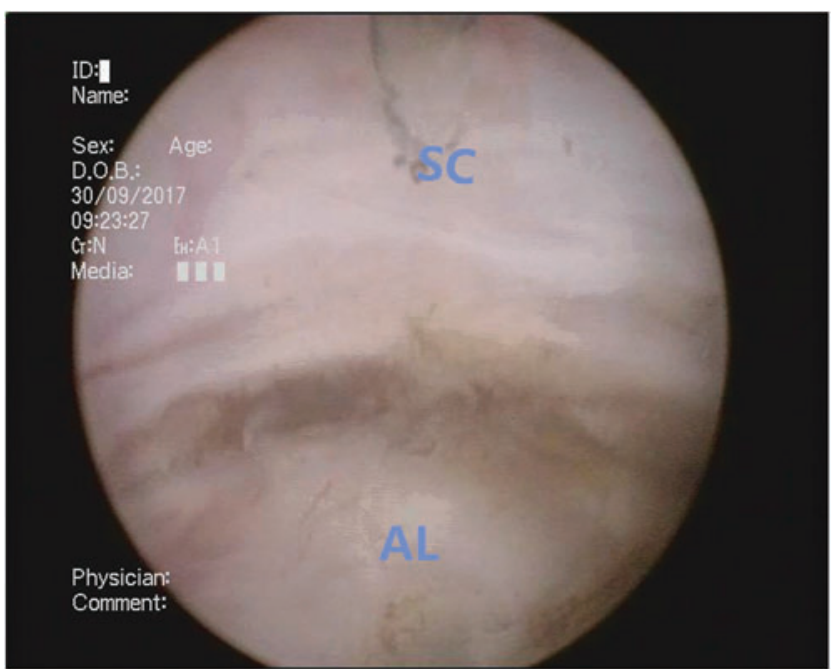

FIG. 6. The anterior lobe (AL) is enucleated along the surgical capsule at the bladder neck.

treatment, the DiLEP group showed markedly reduced blood loss, shorter operation time, faster bladder irrigation and catheterization, and decreased hospitalization duration.

All 152 cases underwent follow-up assessment at 3,6, and 12 months postoperation. Treatment outcomes are listed in Table 3. IPSS, QoL, Qmax, and PVR were comparable in both groups $(P>0.05)$.

No bladder injury, blood transfusion, and TURS cases were noted in this study. None of the cases had long-term urinary incontinence; five (6.6\%) and three (3.9\%) patients had transient incontinence in the DiLEP and PKRP groups, respectively. Secondary bleeding after 1 month was observed in two $(2.6 \%)$ patients in the DiLEP group and in one $(1.3 \%)$ patient in the PKRP group, respectively. Secondary urethral stricture after operation was observed in one (1.3\%) and two (2.6\%) patients in the DiLEP and PKRP groups, respectively. All complications in both groups are shown in Table 4.

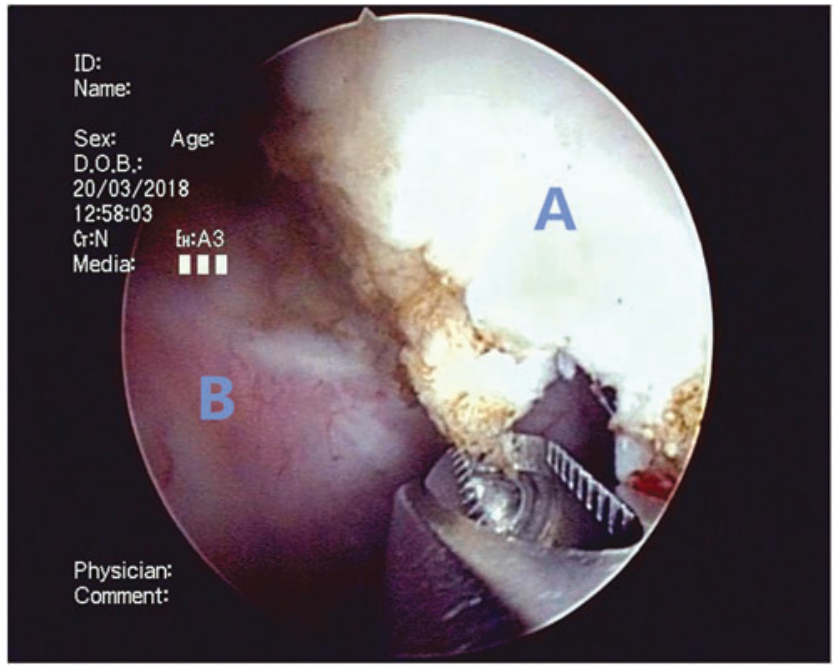

FIG. 7. The adenomas (A) in the bladder were removed by the morcellator system.
Table 1. Baseline Patient Characteristics

\begin{tabular}{lccc}
\hline Parameters & DiLEP $(76)$ & PKRP $(76)$ & $\mathrm{P}$ \\
\hline Age (years) & $73.7 \pm 8.4$ & $71.5 \pm 8.9$ & 0.116 \\
Prostate & $(56-92)$ & $(55-93)$ & \\
$\quad$ volume (mL) & $56.2 \pm 11.9$ & $55.5 \pm 13.1$ & 0.746 \\
PVR (mL) & $204.6 \pm 190)$ & $(34-80)$ & \\
& $(20-700)$ & $(29.5 \pm 186.8$ & 0.867 \\
Qmax (mL/second) & $5.7 \pm 2.2$ & $6.2 \pm 2.5$ & 0.194 \\
& $(1.7-11.5)$ & $(2.5-13.6)$ & \\
IPSS & $24.5 \pm 3.2$ & $25.2 \pm 3.0$ & 0.164 \\
& $(18-30)$ & $(16-31)$ & \\
QoL & $4.7 \pm 0.7$ & $4.9 \pm 0.7$ & 0.115 \\
& $(3-6)$ & $(3-6)$ & \\
\hline
\end{tabular}

Data are represented as mean $\pm \mathrm{SD}$ for each parameter with $n=76$.

DiLEP $=$ diode laser enucleation of the prostate; IPSS $=$ International Prostate Symptom Score; PKRP = plasmakinetic resection of the prostate; $\mathrm{PVR}=$ postvoid residual; $\mathrm{Qmax}=\operatorname{maximum}$ flow rate; $\mathrm{QoL}=$ quality of life; $\mathrm{SD}=$ standard deviation.

\section{Discussion}

TURP remains the gold standard for treating BPH in male individuals with a prostate size between 30 and $80 \mathrm{~mL} .^{12}$ Despite the high success rate of TURP, there are concerns regarding perioperative morbidity and perioperative safety, especially related to bleeding. ${ }^{1}$ Indeed, TURP is not always safe, as cases with prostate volumes exceeding $80 \mathrm{~mL}$ and those with ongoing anticoagulation are totally contraindicated. Therefore, urologists make efforts to search for new endoscopic treatment options for patients with symptomatic BPH. Prostate artery embolization (PAE) is a new treatment for extremely enlarged BPH. PAE provides clinically and statistically significant improvement of symptoms and QoL. ${ }^{13}$ Aquablation is also a novel therapy using a highvelocity waterjet and real-time ultrasound imaging with robotic assistance for targeted removal of the prostate tissue. ${ }^{14}$ Transurethral enucleation of the prostate (TUEP) represents an endoscopic alternative for treating BPH in men, even for cases with large prostates. ${ }^{15}$ With development of laser devices, several laser types, including holmium, KTP, thulium, and diode lasers, have been adapted in TUEP. Holmium laser

Table 2. Perioperative Data

\begin{tabular}{lccc}
\hline Parameters & DiLEP (76) & PKRP (76) & $\mathrm{P}$ \\
\hline $\begin{array}{l}\text { Operative time } \\
\quad \text { minutes) }\end{array}$ & $61.3 \pm 19.0$ & $94.5 \pm 31.5$ & $<0.001$ \\
Hemoglobin & $(35-155)$ & $(35-180)$ & \\
$\quad$ decrease $(\mathrm{g} / \mathrm{L})$ & $9.5 \pm 5.5$ & $12.6 \pm 7.2$ & 0.004 \\
Sodium decrease & $(1-25)$ & $(2-30)$ & \\
$\quad($ mmol/L) & $(0-6)$ & $2.2 \pm 1.6$ & 0.380 \\
Resected weight (g) & $34.8 \pm 13.8$ & $33.2 \pm 12.7$ & 0.448 \\
& $(10-65)$ & $(13-61)$ & \\
Bladder irrigation & $15.9 \pm 11.0$ & $33.2 \pm 21.2$ & $<0.001$ \\
$\quad$ iime (hours) & $(0-83)$ & $(16-97)$ & \\
Catheter duration & $3.1 \pm 1.2$ & $5.5 \pm 1.1$ & $<0.001$ \\
$\quad$ (days) & $(1-6)$ & $(3-8)$ & \\
Hospital & $7.9 \pm 1.1$ & $9.5 \pm 1.1$ & $<0.001$ \\
$\quad$ stay (days) & $(5-10)$ & $(8-12)$ & \\
\hline
\end{tabular}

Data are represented as mean \pm SD for each parameter with $n=76$. 
Table 3. Data at Baseline and Clinical Outcomes at 3, 6, and 12 Months After Surgery

\begin{tabular}{|c|c|c|c|c|}
\hline & Baseline & 3 Months & 6 Months & 12 Months \\
\hline \multicolumn{5}{|l|}{ IPSS } \\
\hline DiLEP & $24.5 \pm 3.2(18-30)$ & $8.4 \pm 2.8(2-14)$ & $6.8 \pm 2.0(2-12)$ & $5.2 \pm 1.9(1-10)$ \\
\hline PKRP & $25.2 \pm 3.0(16-31)$ & $8.7 \pm 2.5(3-15)$ & $7.1 \pm 2.1(2-14)$ & $5.4 \pm 1.6(2-10)$ \\
\hline$P$ & 0.164 & 0.430 & 0.363 & 0.377 \\
\hline \multicolumn{5}{|l|}{ QoL } \\
\hline DiLEP & $4.7 \pm 0.7(3-6)$ & $1.6 \pm 0.7(0-3)$ & $1.4 \pm 0.8(0-3)$ & $1.2 \pm 0.7(0-3)$ \\
\hline PKRP & $4.9 \pm 0.7(3-6)$ & $1.8 \pm 0.7(0-3)$ & $1.5 \pm 0.8(0-3)$ & $1.4 \pm 0.7(0-3)$ \\
\hline$P$ & 0.115 & 0.199 & 0.487 & 0.264 \\
\hline \multicolumn{5}{|l|}{ Qmax } \\
\hline DiLEP & $5.7 \pm 2.2(1.7-11.5)$ & $17.6 \pm 5.3(7.3-37.3)$ & $20.1 \pm 3.9(14.2-36.2)$ & $20.7 \pm 3.8(15.3-37.5)$ \\
\hline PKRP & $6.2 \pm 2.5(2.5-13.6)$ & $18.2 \pm 4.8(6.3-33.2)$ & $21.1 \pm 3.6(15.1-33.0)$ & $21.6 \pm 3.7(15.5-33.5)$ \\
\hline$P$ & 0.194 & 0.475 & 0.123 & 0.144 \\
\hline \multicolumn{5}{|l|}{ PVR } \\
\hline DiLEP & $204.6 \pm 191.1(20-700)$ & $18.6 \pm 17.1(0-50)$ & $17.3 \pm 12.4(0-40)$ & $11.6 \pm 9.4(0-30)$ \\
\hline PKRP & $199.5 \pm 186.8(20-650)$ & $20.6 \pm 17.1(0-55)$ & $18.8 \pm 12.9(0-35)$ & $12.7 \pm 11.0(0-45)$ \\
\hline$P$ & 0.867 & 0.478 & 0.463 & 0.527 \\
\hline
\end{tabular}

Data are represented as mean \pm SD for each parameter with $n=76$.

enucleation of the prostate (HoLEP) provides functional benefits lasting longer than those obtained with TURP or open prostatectomy in large prostates. ${ }^{16,17}$ However, HoLEP has a longer learning curve. Naspro and coworkers suggested $>200$ endoscopic surgeries are required for a surgeon to perform best. ${ }^{18}$ TUEP using a KTP laser or thulium laser is also efficient in symptomatic BPH. ${ }^{19,20}$

Recent technologic advances in laser vaporization of the prostate comprise the development of diode laser systems. Diode lasers have different wavelengths such as 940, 980, 1318, and $1470 \mathrm{~nm}$. Evidence indicates that diode lasers constitute a novel, safe, and efficient technology for treating patients with BPH as TURP/TUEP. DiLEP is advantageous in that it provides markedly reduced blood loss, reduced hospital stay, and decreased catheter indwelling time. ${ }^{21,22}$

The current study showed that no patients required blood transfusion in either of the treatment groups. The procedure of DiLEP is associated with reduced intraoperative hemorrhage reflected by less pronounced postoperative hemoglobin level reduction in comparison with PKRP $(9.5 \pm 5.5 \mathrm{vs}$ $12.6 \pm 7.2, P=0.004)$. The $1470 \mathrm{~nm}$ diode laser beam is ab-

Table 4. Perioperative Complications

\begin{tabular}{lccc}
\hline Complications & $\begin{array}{c}\text { DiLEP (76) } \\
\mathrm{n}(\%)\end{array}$ & $\begin{array}{c}\text { PKRP (76) } \\
\mathrm{n}(\%)\end{array}$ & $\mathrm{P}$ \\
\hline $\begin{array}{l}\text { Intraoperative } \\
\text { Blood transfusion }\end{array}$ & 0 & 0 & - \\
$\quad$ TURS & 0 & 0 & - \\
$\quad$ Capsule perforation & 0 & $1(1.3)$ & 0.316 \\
$\quad$ Bladder injury & 0 & 0 & - \\
Postoperative & & & \\
$\quad$ Secondary bleeding & $2(2.6)$ & $1(1.3)$ & 0.560 \\
$\quad$ Recatheterization & $3(3.9)$ & $2(2.6)$ & 0.649 \\
$\quad$ Transitory stress & $5(6.6)$ & $3(3.9)$ & 0.468 \\
$\quad$ incontinence & & & \\
$\quad \begin{array}{l}\text { Urethral stricture } \\
\text { Irritative symptoms }\end{array}$ & $12(1.3)$ & $2(2.6)$ & 0.560 \\
\hline
\end{tabular}

TURS $=$ transurethral resection syndrome. sorbed by both water and hemoglobin, which results in excellent coagulation and rapid vaporization of the tissue. In the current study, surgical times for DiLEP and PKRP were $61.3 \pm 19.0$ and $94.5 \pm 31.5$ minutes, indicating a stark reduction in the former procedure $(P<0.001)$. DiLEP was faster in $\mathrm{BPH}$ treatment than PKRP, with a dramatically shortened operation time, which reduces surgical risks, especially in patients with cardiopulmonary insufficiency disease.

TURS is another severe complication of traditional TURP; however, no TURS case was observed in either of the treatment groups, with comparable serum sodium reductions in both groups. Because physiologic saline was used for irrigation, DiLEP and PKRP almost had no risk of TURS. In addition, rapid vaporization of the tissue and coagulation occurred almost at the same time during DiLEP for BPH, which to a great extent reduced the exposure time of blood vessels and subsequent odds of irrigation water entering into circulation. $^{23}$

Transient stress urinary incontinence is often encountered after endosurgery for prostate enucleation, and was found in $3.3 \%-7.5 \%$ cases after DiLEP. ${ }^{24}$ This study demonstrated that DiLEP occurrence was $6.6 \%$, compared with $3.9 \%$ in the PKRP group $(P=0.468)$, similar to HoLEP and thulium laser vaporization enucleation of the prostate (ThuVEP). ${ }^{25,26}$ Stress urinary incontinence disappeared at 3 months postoperatively by exercising the levator ani muscle. According to our experience, prostatic apexes are critical during DiLEP surgery for large prostates. Best efforts should be made to reduce injuries of the external sphincter during enucleation.

The incidence of irritative symptoms in DiLEP was $15.8 \%$, with no significant difference in comparison with the PKRP group $(15.8 \%$ vs $9.2 \%, P=0.220)$. Such finding could be explained by use of the $1470 \mathrm{~nm}$ diode laser, which penetrates as deep as $2.3 \mathrm{~mm}$, with elevated coagulation depth, as found in TURP. ${ }^{27}$ In contrast to other lasers, less coagulated and necrotic tissues after DiLEP are removed postoperatively. ${ }^{21}$ The incidence rates of recatheterization and urethral stricture after DiLEP were $3.9 \%$ and $1.3 \%$, respectively, corroborating Razzaghi and coworkers, ${ }^{28}$ with both groups showing comparable values. 
Accordingly, the curative effects of DiLEP and PKRP are similar; indeed, significant improvements were obtained in both groups from baseline values in IPSS, QoL score, Qmax, and PVR at postoperative 3, 6, and 12 months. However, both groups showed similar values for various assessment parameters at these time points. The present study suggested that DiLEP is as safe and efficient as PKRP.

This study had limitations because of the small sample size and short follow-up. Therefore, the current findings require confirmation in large prospective randomized trials.

\section{Conclusions}

Both DiLEP and PKRP are safe and efficient for BPH, as shown in this initial study. In comparison with PKRP, DiLEP reduces the risk of hemorrhage, takes less time, decreases bladder irrigation and catheterization times, and results in decreased hospitalization duration. Further well-designed, prospective randomized studies with prolonged follow-up, including large numbers of patients, are required to confirm the benefits of DiLEP in symptomatic BPH.

\section{Acknowledgments}

The authors acknowledge financial support from the Guiding Medical Project of Shanghai Science and Technology Committee (Grant No. 16411972000) and the Shanghai Key Medical Specialty Program (Grant No. ZK2015B04).

\section{Author Disclosure Statement}

The authors declare that they have no competing interests.

\section{References}

1. Rassweiler J, Teber D, Kuntz R, Hofmann R. Complications of transurethral resection of the prostate (TURP) Incidence, management and prevention. Eur Urol 2006;50: 969-980.

2. Ahyai SA, Gilling P, Kaplan SA, Kuntz RM, Madersbacher S, Montorsi F, Speakman MJ, Stief CG. Meta-analysis of functional outcomes and complications following transurethral procedures for lower urinary tract symptoms resulting from benign prostatic enlargement. Eur Urol 2010;58: 384-397.

3. Bachmann A, Woo HH, Wyler S. Laser prostatectomy of lower urinary tract symtoms due to benign prostate enlargement: A critical review of evidence. Curr Opin Urol 2012;22:22-33.

4. Fraundorfer MR, Gilling PJ. Holmium: YAG laser enucleation of the prostate combined with mechanical morcellation: Preliminary results. Eur Urol 1998;33:69-72.

5. Gilling PJ, Wilson LC, King CJ, Westenberg AM, Frampton CM, Fraundorfer MR. Long-term results of a randomized trial comparing holmium laser enucleation of the prostate and transurethral resection of the prostate: Results at 7 years. BJU Int 2012;109:408-411.

6. Hauser S, Rogenhofer S, Ellinger J, Strunk T, Müller SC, Fechner G. Thulium laser (Revolix) vapoenucleation of the prostate is a safe procedure in patients with an increased risk of hemorrhage. Urol Int 2012;88:390-394.

7. Thangasamy IA, Chalasani V, Bachmann A, Woo HH. Photoselective vaporisation of the prostate using $80-\mathrm{W}$ and $120-\mathrm{W}$ laser versus transurethral resection of the prostate for benign prostatic hyperplasia: A systematic review with meta-analysis from 2002 to 2012. Eur Urol 2012;62:315323.

8. Yang KS, Seong YK, Kim IG, Han BH, Kong GS. Initial experiences with a $980 \mathrm{~nm}$ diode laser for photoselective vaporization of the prostate for the treatment of benign prostatic hyperplasia. Korean J Urol 2011;52:752-756.

9. Wendt-Nordahl G, Huckele S, Honeck P, Alken P, Knoll T, Michel MS, Häcker A. 980-nm Diode laser: A novel laser technology for vaporization of the prostate. Eur Urol 2007; 52:1723-1728.

10. Seitz M, Sroka R, Gratzke C, et al. The diode laser: A novel side-firing approach for laser vaporisation of the human prostate-Immediate efficacy and 1-year follow-up. Eur Urol 2007;52:1717-1722.

11. Gratzke C, Bachmann A, Descazeaud A, et al. EAU guidelines on the assessment of non-neurogenic male lower urinary tract symptoms including benign prostatic obstruction. Eur Urol 2015;67:1099-1109.

12. Madersbacher S, Alivizatos G, Nordling J, Sanz CR, Emberton M, de la Rosette JJ. EAU 2004 guidelines on assessment, therapy and follow-up of men with lower urinary tract symptoms suggestive of benign prostatic obstruction (BPH Guidelines). Eur Urol 2004;46:547-554.

13. Ray AF, Powell J, Speakman MJ, et al. The UK ROPE Study: Efficacy and safty of prostate artery embolisation for benign prostatic hyperplasia. An observational study and propensity matched comparison with transurethral resection of the prostate. BJU Int 2018;122:270-280.

14. Gilling $\mathrm{P}$, Reuther R, Kahokehr A, Fraundorfer $M$. Aquablation-Image-guided robot-assisted waterjet ablation of the prostate: Initial clinical experience. BJU Int 2016;117:923-929.

15. Geavlete B, Stanescu F, Iacoboaie C, Geavlete P. Bipolar plasma enucleation of the prostate vs open prostatectomy in large benign prostatic hyperplasia cases-A medium term, prospective, randomized comparison. BJU Int 2013;111: 793-803.

16. Gilling PJ, Wilson LC, King CJ, et al. Long-term results of a randomized trail comparing holmium laser enucleation of the prostate and transurethral resection of the prostate: Results at 7 years. BJU Int 2011;109:408-411.

17. Kuntz RM, Lehrich K, Ahyai SA. Holmium laser enucleation of the prostate versus open prostatectomy for prostates greater than 100 grams: 5-Year follow-up results of a randomised clinical trial. Eur Urol 2008;53: 160-166.

18. Naspro R, Suardi N, Salonia A, et al. Holmium laser enucleation of the prostate versus open prostatectomy for prostates >70 g: 24-Month follow-up. Eur Urol 2006;50: 563-568.

19. Capitán C, Blázquez C, Martin MD, Hernández V, de la Peña E, Llorente C. Greenlight HPS 120-W laser vaporization versus transurethral resection of the prostate for the treatment of lower urinary tract symptoms due to benign prostatic hyperplasia: A randomized clinical trial with 2year follow-up. Eur Urol 2011;60:734-739.

20. Cui D, Sun F, Zhuo J, et al. A randomized trial comparing thulium laser resection to standard transurethral resection of the prostate for symptomatic benign prostatic hyperplasia: Four-year follow-up results. World J Urol 2014;32: 683-689.

21. $\mathrm{Xu} \mathrm{A,} \mathrm{Zou} \mathrm{Y,} \mathrm{Li} \mathrm{B,} \mathrm{et} \mathrm{al.} \mathrm{A} \mathrm{randomized} \mathrm{trial} \mathrm{comparing}$ diode laser enucleation of the prostate with plasmakinetic enucleation and resection of the prostate for the treatment 
of benign prostatic hyperplasia. J Endourol 2013;27:12541260.

22. Wu G, Hong Z, Li C, Bian C, Huang S, Wu D. A comparative study of diode laser and plasmakinetic in transurethral enucleation of the prostate for treating large volume benign prostatic hyperplasia: A randomized clinical trial with 12month follow-up. Lasers Med Sci 2016;31:599-604.

23. Zhao Y, Liu C, Zhou G, Yu C, Zhang Y, Ouyang Y. A retrospective evaluation of benign prostatic hyperplasia treatment by transurethral vaporization using a $1470 \mathrm{~nm}$ laser. Photomed Laser Surg 2013;31:626-629.

24. Lusuardi L, Mitterberger M, Hruby S, Kunit T, Kloss B, Engelhardt PF, Sieberer M, Janetschek G. Update on the use of diode laser in the management of benign prostate obstruction in 2014. World J Urol 2015;33:555-562.

25. Yin L, Teng J, Huang C-J, Zhang X, Xu D. Holmium laser enucleation of the prostate versus transurethral resection of the prostate: A systematic review and meta-analysis of randomized controlled trials. J Endourol 2013;27:604-611.

26. Netsch C, Pohlmann L, Herrmann TR, et al. 120W 2microm thulium: Yttrium-aluminium-garnet vapoenucleation of the prostate: 12-Month follow-up. BJU Int 2012; 110:96-101.

27. Seitz M, Ruszat R, Bayer T, Tilki D, Bachmann A, Stief C, Sroka R, Reich O. Ex vivo and in vivo investigations of the novel $1470 \mathrm{~nm}$ diode laser for potential treatment of benign prostatic enlargement. Lasers Med Sci 2009;24:419-424.

28. Razzaghi MR, Mazloomfard MM, Mokhtarpour H, Moeini A. Diode laser $(980 \mathrm{~nm})$ vaporization in comparison with transurethral resection of the prostate for benign prostatic hyperplasia: Randomized clinical trial with 2-year followup. Urology 2014;84:526-532.
Address correspondence to:

Guowei Shi, MD

Department of Urology

The Fifth People's Hospital of Shanghai

Fudan University

801 Heqing Road

Minhang District

Shanghai 200240

P.R. China

E-mail: dr.sgw@189.cn

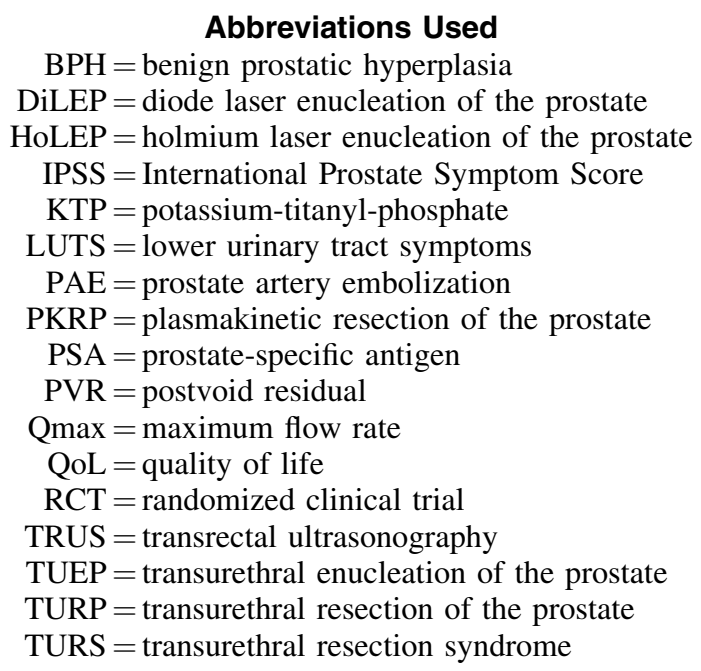

